\title{
Recent Advances In The Physics Of Collective Excitations In The Paul Trap Simulator Experiment
}

\author{
E. P. Gilson ${ }^{\mathrm{a}}$, M. Chung ${ }^{\mathrm{a}}$, R. C. Davidson ${ }^{\mathrm{a}}$, M. Dorf ${ }^{\mathrm{a}}$, P. C. Efthimion $^{\mathrm{a}}$, A. B. Godbehere ${ }^{\mathrm{b}}$, \\ R. Majeski ${ }^{\mathrm{a}}$ \\ ${ }^{a}$ Princeton Plasma Physics Laboratory, Princeton, New Jersey, USA \\ ${ }^{b}$ Cornell University, Ithaca, New York, USA
}

\begin{abstract}
The Paul Trap Simulator Experiment (PTSX) is a compact laboratory linear Paul trap that simulates the transverse dynamics of a charged-particle bunch coasting through a magnetic alternating-gradient $(\mathrm{AG})$ transport system. The transverse dynamics of particles in the AG system in the beam's frame-of-reference and those of particles in PTSX are described by the same sets of equations, including all nonlinear space-charge effects. Initial experimental results are presented in which the collective transverse symmetric mode $(m=0)$ and quadrupole mode $(m=2)$ have been observed in pure-barium-ion plasmas in PTSX, with an initial depressed-tune $\nu / \nu_{0} \sim 0.9$, with the intent of identifying collective modes whose signature will serve as a robust diagnostic for key properties of the beam, such as line density and transverse emittance. The results of particle-in-cell simulations performed with the WARP code are compared to the experimental data.
\end{abstract}

Key words: Ion Beam, Accelerator, Plasma, Paul Trap

PACS: 29.27.-a, 41.85.Ja, 52.27.Jt

\section{Introduction}

One-component nonneutral plasmas and intense charged-particle beams support a wide variety of collective oscillations [1-4] that depend on the details of the distribution function, the focusing field strength, the self-field intensity parameter $\hat{s}$, and geometric effects such as the proximity of the conducting wall. The focusing field strength is characterized by the average transverse oscillation frequency $\omega_{q}$ of particles moving through the focusing system, Preprint submitted to Nuclear Instruments and Methods in Physics Research A

July 24, 2008 
and the plasma is characterized by the plasma frequency $\omega_{p}$. The normalized intensity parameter is then defined as $\hat{s}=\omega_{p}^{2} / 2 \omega_{q}^{2}$ and describes the relative strength of the self-fields of the particles as compared to the strength of the focusing field. The frequencies of the collective oscillations typically involve various combinations of the frequencies $\omega_{q}, \omega_{p}$, and $\left(\omega_{q}^{2}-\omega_{p}^{2} / 2\right)^{1 / 2}[1]$, modified by geometric effects $\left(r_{p} / r_{w}\right)$. This paper presents the results of experiments on the Paul Trap Simulator Experiment (PTSX) that investigate the detailed properties of collective excitations that occur naturally as the result of beam mismatch and imperfections in the voltage waveform.

As high-intensity beams continue to become increasingly relevant and desirable in areas such as high energy and nuclear physics, ion-beam-driven high energy density physics, heavy ion fusion, and spallation neutron sources $[1-3,5-7]$, it is important to develop an improved experimental and theoretical understanding of these systems. In the present work, in addition to testing and validating theoretical models of collective oscillations in intense charged-particle beams [1-4, 8-15], particular emphasis is placed on identifying collective modes whose signature will serve as a robust diagnostic for key properties of the beam, such as line density and transverse emittance.

\section{The Paul Trap Simulator Experiment}

The Paul Trap Simulator Experiment (PTSX) is a compact and flexible laboratory facility that simulates the propagation of intense charged-particle beams over thousands of lattice periods through magnetic alternating-gradient (AG) quadrupole transport systems [16-24]. The simulation makes use of the isomorphism between the transverse equations of motion for particles in the two systems [16, 25-27]. The isomorphism exists because PTSX can be thought of as an experiment performed in a charged-particle beam's frame of reference.

The PTSX device is a linear Paul trap [28] confining a one-component plasma of particles with charge $e_{b}$, where the $e_{b} E_{\perp}^{e x t}$ forces that the PTSX electrodes exert on the trapped plasma particles are analogous to the $e_{b} v_{z} \times B_{\perp}^{e x t}$ forces that the $\mathrm{AG}$ system exert on the beam particles in the beam frame provided that long, coasting beams that are thin relative 
to the AG system magnet spacing are considered. Specifically, the amplitude and frequency of the voltage waveform applied to the PTSX electrodes correspond to the quadrupole magnet strength and lattice spacing in the $\mathrm{AG}$ system. In addition to the equivalence of the applied forces, the self-field forces in both systems can be described by scalar potentials that obey Poisson's equation. In Ref. [16], it was shown that the self-consistent transverse Hamiltonians and the resulting Vlasov equations for the AG system and the PTSX system are equivalent, neglecting end effects. Thus, the very good confinement properties of ions in PTSX and the arbitrary form of the voltage waveform applied to the confining electrodes make PTSX a useful laboratory facility in which to simulate transverse beam compression in an AG system.

\section{PTSX Apparatus}

As shown in Fig. 1, the PTSX device is a linear Paul trap constructed from a 2.8 m-long, $r_{w}=10 \mathrm{~cm}$-radius cylinder. The cylinder is divided into two $40 \mathrm{~cm}$-long end cylinders and a $2 L=2$ m-long central cylinder. All cylinders are azimuthally divided into four $90^{\circ}$ segments so that when an oscillating voltage $V_{0}(t)$ is applied with alternating polarity on adjacent segments, the resulting oscillating transverse quadrupole electric field exerts a ponderomotive force that confines the plasma radially. To trap the plasma axially, the two end cylinders are biased to a constant voltage $\hat{V}$. Voltage waveforms with amplitudes up to $400 \mathrm{~V}$ and frequencies up to $100 \mathrm{kHz}$ can be used. The trapping voltage is nominally $\hat{V}=36 \mathrm{~V}$. The vacuum pressure of $5 \times 10^{-9}$ Torr prevents neutral collisions from playing an important role in the plasma behavior.

The plasma source for these experiments is a $1.3 \mathrm{~cm}$-diameter atomic oven loaded with barium. Solid barium is loaded into the $10 \mathrm{~cm}$-long tube and packed against the closed end. The open end of the tube is plugged with platinum mesh that serves as the ionizer material. Resistive band heaters, placed at the two ends of the tube, heat the barium to near 700 $\mathrm{C}$ and heat the platinum mesh ionizer to $1100 \mathrm{C}$. The neutral barium vapor, created by the heating, passes through the platinum mesh and becomes ionized through the process of contact ionization. The ions are extracted by applying a bias of up to 80 volts between the 


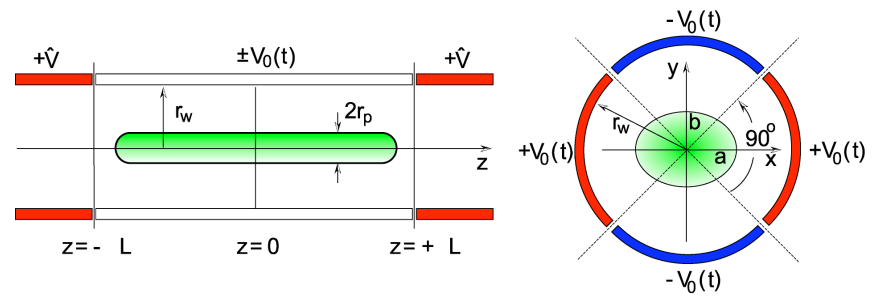

Figure 1: The PTSX device consists of three cylindrical electrodes with radius $r_{w}=0.1 \mathrm{~m}$, each divided into four $90^{\circ}$ sectors. An oscillating voltage $\pm V_{0}(t)$ confines the charge bunch in the transverse plane to a radius $r_{p}$. Static voltages $+\hat{V}$ on the end electrodes confine the ions axially within a length $2 L=2 \mathrm{~m}$.

ionizer and an acceleration grid. The ions then pass through a separately biased deceleration grid. The ion source is situated in the middle of one of the $40 \mathrm{~cm}$-long cylinders, and to inject a barium ion plasma into the trap, the segments on this $40 \mathrm{~cm}$-long cylinder are temporarily set to oscillate with the voltage $\pm V_{0}(t)$.

Although not the case in the present work, in many PTSX experiments, it is desirable to create plasma that is trapped axially. In this case, the injection time $t_{i}$ is several milliseconds in order to allow barium ions to fill the trap. The $40 \mathrm{~cm}$-long cylinder is then set to the trapping voltage $+\hat{V}$. After trapping the plasma for a time $t_{t}$, that can be up to $300 \mathrm{~ms}$, the $40 \mathrm{~cm}$-long cylinder on the opposite end of the PTSX device from the ion source is set to oscillate with voltage $\pm V_{0}(t)$, and the plasma streams out of the trap. Part of the exiting plasma is collected on a moveable $5 \mathrm{~mm}$-diameter collector disk. The inject-trap-dump cycle is repeated to reduce the uncertainty in the data. The collector is moved along a null in the applied potential in the transverse plane in order to collect a radial charge profile of the trapped plasma.

The experimental results reported herein were obtained while using PTSX in a passthrough mode without any axial trapping. The pass-through mode is a steady-state mode where the injection and dumping electrodes are set to always oscillate with the voltage waveform $\pm V_{0}(t)$ that is applied to the two-meter-long electrodes. The ions are emitted from the ion source, travel the length of the machine, and are dumped out the end. Rather than the collector disk, a capacitive pickup electrode, described below, is used to measure the steady-state oscillation frequencies of the plasma for these experiments. 


\section{Collective Excitations}

The following discussion closely follows that in Davidson and Qin [1]. For the KapchinskijVladimirskij (KV) distribution, describing a uniform-density, mono-energetic beam, the coupled envelope equations for the transverse dimensions of the beam $a(s)$ and $b(s)$ are written as:

$$
\begin{gathered}
\frac{d^{2}}{d s^{2}} a(s)+\kappa_{x}(s) a(s)-\frac{2 K_{b}}{a(s)+b(s)}=\frac{\epsilon_{x}^{2}}{a^{3}(s)} \\
\frac{d^{2}}{d s^{2}} b(s)+\kappa_{y}(s) b(s)-\frac{2 K_{b}}{a(s)+b(s)}=\frac{\epsilon_{y}^{2}}{b^{3}(s)}
\end{gathered}
$$

where $K_{b}$ is the beam perveance and $\epsilon_{x}$ and $\epsilon_{y}$ are the transverse emittances, and $s=\beta c t$. For simplicity, $\epsilon_{x}=\epsilon_{y}=\epsilon=$ const. here. In the smooth focusing model, the lattice functions that describe the periodic focusing of the AG system, $\kappa_{x}(s)$ and $\kappa_{y}(s)$, are both replaced by $\kappa_{s f}=\omega_{q}^{2} / \beta^{2} c^{2}$ which is independent of $s$. In a smooth focusing equilibrium for a matched beam, $a(s)=b(s)=r_{b}$ where $r_{b}$ satisfies

$$
\kappa_{s f} r_{b}-\frac{K_{b}}{r_{b}}=\frac{\epsilon^{2}}{r_{b}^{3}} .
$$

The linear mode frequencies are determined by substituting $a(s)=r_{b}+\delta a(s)$ and $b(s)=$ $r_{b}+\delta b(s)$ into Eqs. (1) and (2) and keeping only the terms linear in $\delta a(s)$ and $\delta b(s)$. Modes with density perturbations of the form $\cos (m \theta)$ with $m=0$ and $m=2$ are found by seeking solutions of the resulting coupled equations of the form $\delta a(s)= \pm \delta b(s)$. In the case where $\delta a(s)=\delta b(s)$, Eq. (1) reduces to

$$
\frac{d^{2}}{d s^{2}} \delta a(s)+\left(\kappa_{s f}+\frac{K_{b}}{r_{b}^{2}}+\frac{3 \epsilon^{2}}{r_{b}^{4}}\right) \delta a(s)=0 .
$$

By noting that the phase advance $\sigma$ can be written as $\sigma=\epsilon S / r_{b}^{2}$, where $S$ is the lattice periodicity, and that the vacuum phase advance can be written as $\sigma_{v}=S \sqrt{\kappa_{s f}}$ when $K_{b}=0$, Eq. (4) can be simplified to become,

$$
\frac{d^{2}}{d s^{2}} \delta a(s)+2\left[\left(\frac{\sigma_{v}}{S}\right)^{2}+\left(\frac{\sigma}{S}\right)^{2}\right] \delta a(s)=0 .
$$

Since $\left(\sigma / \sigma_{v}\right)^{2}=1-\hat{s}$ for a uniform density beam, and since $s=\beta c t$, the angular frequency of the mode can finally be written as,

$$
\omega_{m=0}^{2}=\omega_{g}^{2}(4-2 \hat{s}) .
$$


This is the mode with $m=0$ and is a body-wave mode in which the beam's size oscillates without any angular dependence. Such as oscillation must be a body-wave, since the beam density must increase and decrease throughout the beam over the oscillation period.

In the case where $\delta a(s)=-\delta b(s)$, a similar analysis leads to,

$$
\omega_{m=2}^{2}=\omega_{q}^{2}(4-3 \hat{s})
$$

This is the mode with $m=2$ and is a quadrupolar surface mode in which the beam envelope has an oscillating elliptical perturbation. This mode is a surface mode because the density in the bulk need not change since the excess charge near the ends of the major axis of the elliptical transverse beam shape is equal to the shortage in charge near the ends of the minor axis of the elliptical transverse beam shape. Note that these mode frequencies, and many more, can also be found by solving the linearized Vlasov-Poisson equation with a KV distribution in order to find the eigenvalues for the cases of surface modes with $\partial / \partial z=0$ and $\partial / \partial \theta \neq 0$, and for body modes with $\partial / \partial z=0$ and $\partial / \partial \theta=0$.

In the absence of space-charge $\left(K_{b}, \hat{s} \rightarrow 0\right)$, the two angular mode frequencies are both equal to $2 \omega_{q}$. As the amount of space-charge in the beam increases, the two mode frequencies both decrease, but at different rates. Therefore, the two mode frequencies become distinct. Simultaneous measurement of both mode frequencies is then a robust measurement of the normalized intensity parameter $\hat{s}$.

\section{Experimental Results}

The detection of the $m=0$ and $m=2$ collective oscillations is achieved with azimuthally segmented ring electrodes that are placed inside the primary confinement electrodes. Modes with different azimuthal mode numbers are detected by appropriately choosing the relative phase of the signals on the azimuthal segments. This technique allows greater coupling to the mode of interest while also discriminating against other modes.

In order to detect collective transverse plasma modes in PTSX, a special diagnostic was designed, built, and installed. The segmented electrodes that provide the transverse 
confinement in PTSX cannot be used simultaneously as diagnostics since the expected submillivolt signal would be dominated by the several-hundred-volt signal applied to confine the ions.

The collective-mode diagnostic is a segmented cylinder that is $20 \mathrm{~cm}$-long and $10 \mathrm{~cm}$ in diameter. This means that the diagnostic is one-tenth the length of the confinement region of the trap and half the diameter. The diameter is small enough to be close to the plasma in order to maximize the strength of the detected signal, but large enough to not intercept the plasma itself. The length is short enough so that the plasma ions do not spend an appreciable amount of time in a region where the applied transverse focusing field is screened-out by the diagnostic.

The diagnostic cylinder (Fig. 2) is made from four, thin, copper sheets that are rolled into quarter-cylinders and held in place against delrin collars. The collars are machined to ensure the precise alignment of, and gap spacing between the diagnostic plates. Adjustable legs with insulating delrin "feet" keep the diagnostic well-centered on the machine axis The diagnostic is placed one quarter of the way along the length of the two-meter-long PTSX primary electrodes in order to prevent it from interfering with ion injection or the field-ofview of the laser-induced fluorescence diagnostic at the center of PTSX.

Each of the four collective-mode diagnostic plates is connected to an op-amp follower in order to buffer the signal. The signals are then sent to 6th-order Chebyshev low pass filters, and then to amplifiers with gains of 100. The lattice frequency $f$ and the average transverse focusing frequency $f_{q}=\omega_{q} / 2 \pi$ are well separated for usual PTSX parameters; in fact, $f_{q} / f=\sigma_{v}^{s f} / 2 \pi \sim 1 / 7$. The PTSX voltage waveform amplitude is set to $150 \mathrm{~V}$ and the frequency is set to $f=60 \mathrm{kHz}$ so that $\omega_{q}=5.0 \times 10^{4} \mathrm{~s}^{-1}$ and $\sigma_{v}=49^{\circ}$. The passband of the filters is therefore chosen to be between 0 and roughly $23 \mathrm{kHz}$. The frequency response of the circuit is shown in Fig. 3. The precision of the values of the circuit's components was not high enough to ensure the proper 6th-order Chebyshev frequency response. Nevertheless, the frequency response is adequate for the present experiment.

The four signals can then be optionally inverted before they are added together. If the four signals are simply added together, the result is a signal that is maximally sensitive 


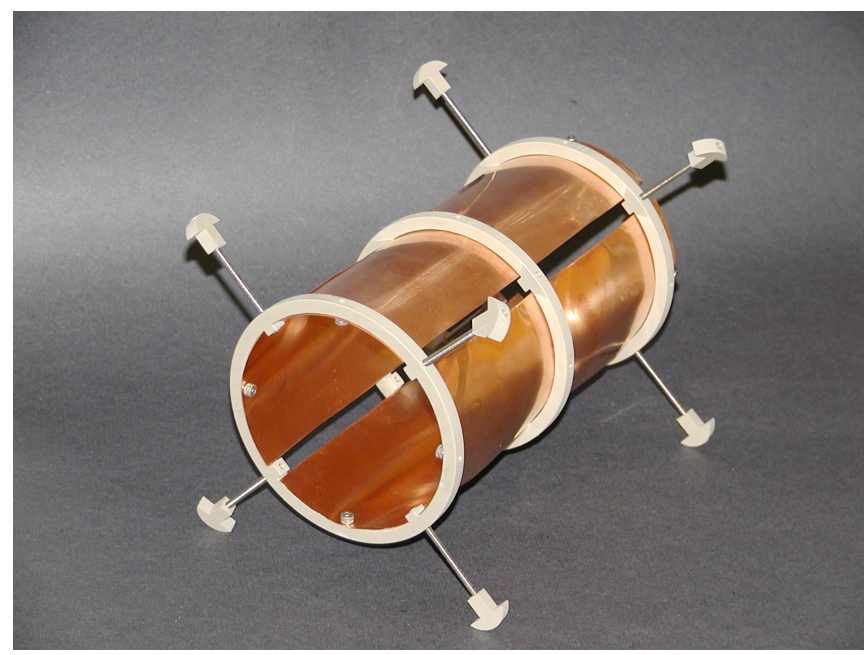

Figure 2: The collective mode diagnostic is a $10 \mathrm{~cm}$-diameter, $20 \mathrm{~cm}$-long cylinder divided into four azimuthal sectors. The sectors are held in place by insulating delrin collars. Threaded rods with delrin "feet" keep the diagnostic centered on the machine axis.

to the $m=0$ symmetric mode, and is relatively insensitive to the $m=2$ quadrupole mode. If, instead, every other channel is inverted before the four channels are added, then the resulting signal contains mostly the $m=2$ mode. This detection method depends on the spatial orientation of the modes being aligned with the orientation of the diagnostic electrodes and is important in helping to properly identify the mode by establishing both the mode's frequency and spatial structure.

The large ion source bias of $15 \mathrm{~V}$ increases the amount of plasma injected, and also increases the equilibrium radius of the plasma, both of which lead to increased coupling of the plasma to the collective-mode diagnostic. It must be noted that the modes that were detected in this experiment were not purposefully excited. The variety and amplitudes of the naturally-occurring modes seen likely depended on the inherent mismatch of the ion beam from the circular ion beam into the fully time-dependent electrostatic confining fields.

Any single sector of the collective-mode diagnostic is sensitive to both the $m=0$ and $m=2$ modes, and so the initial experiments were performed with only one sector. The resulting frequency power spectrum is shown in Figure 4. This final spectrum is obtained by dividing the measured spectrum by the transfer function of the circuit as shown in Fig. 3. 


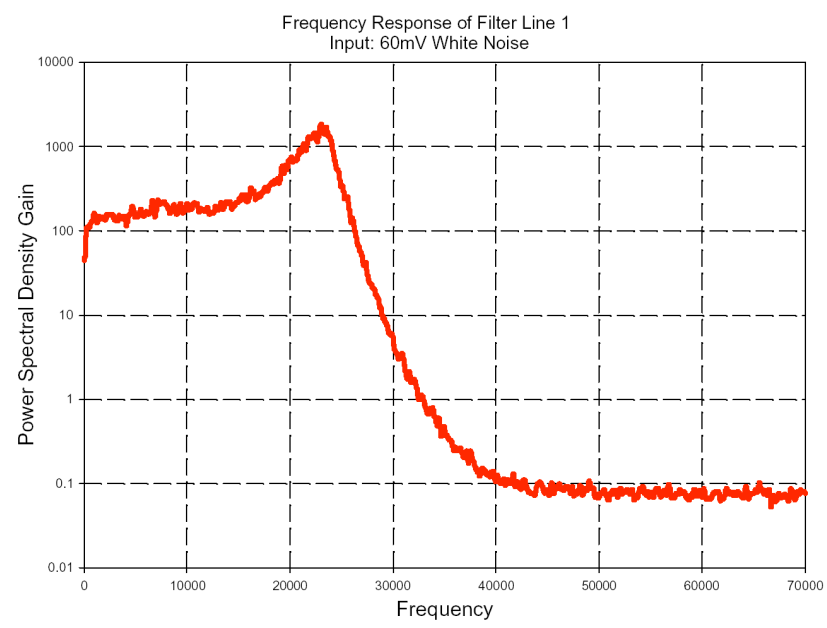

Figure 3: The frequency response of the filter/amplifier circuit is measured by driving the circuit with 60 $\mathrm{mV}$ white noise. The passband covers frequencies from 0 to roughly $23 \mathrm{kHz}$.

This division compensates for the small non-uniformity in the gain of the circuit in the pass-band below $30 \mathrm{kHz}$. Prominent features of the data include: a peak at $7.5 \mathrm{kHz}$, a pair of peaks near $15 \mathrm{kHz}$, and a peak at $19.6 \mathrm{kHz}$. The main interest is in the resolved pair of peaks near $15 \mathrm{kHz}$. The peaks in the spectrum shown in Fig. 4 all disappear when the plasma injection is stopped, demonstrating that the peaks are not direct coupling to subharmonics of the $60 \mathrm{kHz}$ lattice oscillation frequency.

For the system parameters used in this experiment, $2 f_{q}=16.0 \mathrm{kHz}$, and so the two peaks near $15 \mathrm{kHz}$ are candidates to be the $m=0$ symmetric mode and the $m=2$ quadrupole mode. It is essential that the normalized intensity parameters $\hat{s}$ corresponding to the mode frequencies' shifts down from $2 f_{q}$ agree with one another. The beam has only one unique normalized intensity parameter at any moment, and the dispersion relations predict two specific values for the frequencies of the $m=0$ and $m=2$ modes. The plot in Figure 5 shows the mode frequencies as a function of $\hat{s}$ over the allowed range of $\hat{s}$ from 0 to unity. There is one, and only one, normalized intensity parameter for which the two modes have precisely the $15.3 \mathrm{kHz}$ and $15.0 \mathrm{kHz}$ frequencies observed in the experiment. In this case, the normalized intensity parameter measured is $\hat{s}=0.15$. Particle-in-cell simulations performed with the WARP code show that there is a negligible frequency shift in the mode frequencies 


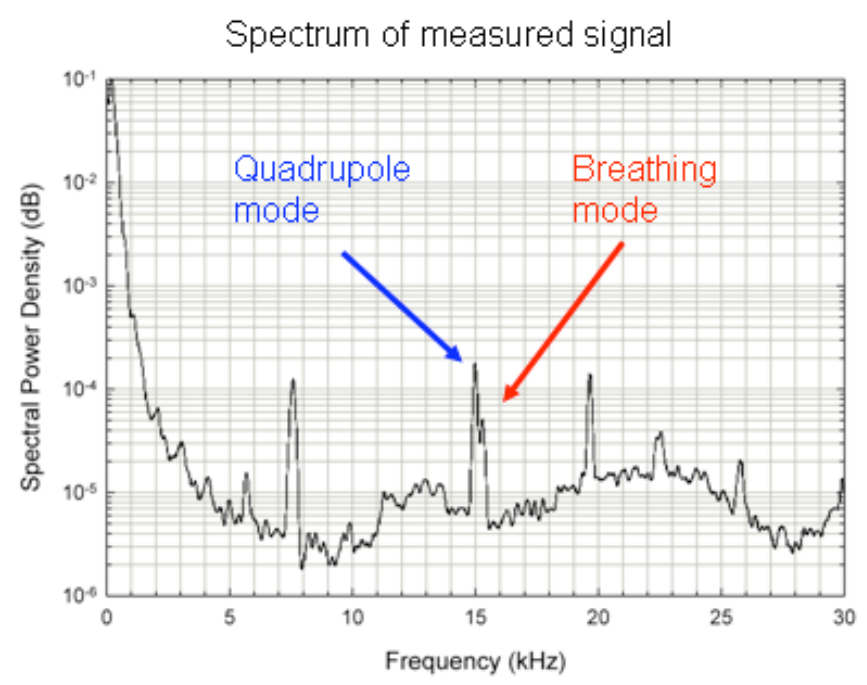

Figure 4: The frequency spectrum of a single plate of the collective mode diagnostic exhibits prominent peaks at 7.5, 15.0, 15.3, and $19.6 \mathrm{kHz}$. The peaks at 15.0 and $15.3 \mathrm{kHz}$ are consistent with the expected frequencies of the $m=0$ and $m=2$ modes. The nature of the other prominent peaks is unknown.

expected from Eqs. (6) and (7) due to the plasma's distribution function not corresponding to the idealized KV distribution assumed in Section 4.

It is known from many previous experiments performed with PTSX that $\hat{s}=0.15$ is a reasonable value of $\hat{s}$ for the experimental conditions used. Measurements of the normalized intensity parameter are usually made with an axially-trapped plasma that is then dumped onto the collector disk. The on-axis measured charge is used to compute the on-axis number density by estimating the volume from which the measured charge came. The measured value of $\hat{s}=0.15$ from the collective-mode diagnostic experiment is reasonable since the ion source bias voltage was high, but PTSX was operated in the pass-through mode. In pass-through mode, the plasma density is expected to be approximately half the density of an axially-trapped plasma.

Planned collective-mode experiments include determining the spatial structure of the plasma oscillations by using all four of the diagnostic electrode plates. It is also important to strengthen the identification of the observed peaks in the spectrum with the $m=0$ and $m=2$ modes by verifying that the frequencies of the peaks scales with $\hat{s}$ as predicted in Eqs. (6) and (7). Finally, a complete understanding of the full spectrum of frequencies in 


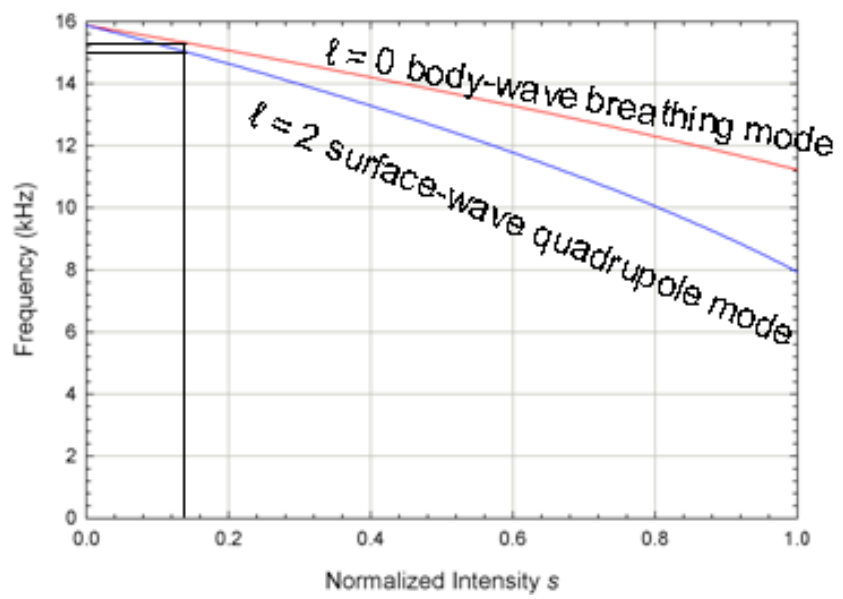

Figure 5: The mode frequencies of the $m=0$ and $m=2$ modes are both $2 f_{q}$ when $\hat{s}=0$ and decrease with $\hat{s}$. However, the $m=2$ quadrupole mode decreases more rapidly, leading to a split in the frequencies. Since the plasma is characterized by a single normalized intensity $\hat{s}$, a simultaneous measurement of the two mode frequencies should correspond to a unique $\hat{s}$.

the measured data will be pursued, including an understanding of the relative amplitudes of the peaks.

\section{Conclusions}

An azimuthally segmented collective mode diagnostic was used in PTSX do detect the naturally occurring symmetric $m=0$ and quadrupole $m=2$ modes in a pure barium plasma. The two mode frequencies are both consistent with a single value of the normalized intensity parameter $\hat{s}=0.15$ that is in agreement with an independent measurement made with a destructive diagnostic. Future experiments will seek to measure the spatial structure of these modes and measure the scaling of the frequencies with the $\hat{s}$.

\section{Acknowledgment}

This research was supported by the U.S. Department of Energy. 


\section{References}

[1] R. C. Davidson and H. Qin, Physics of Intense Charged Particle Beams in High Intensity Accelerators (World Scientific, Singapore, 2001).

[2] A. W. Chao, Physics of Collective Beam Instabilities in High Energy Accelerators (Wiley, New York, 1993).

[3] M. Reiser, Theory and Design of Charged Particle Beams (Wiley, New York, 1994).

[4] R. C. Davidson, Physics of Nonneutral Plasmas (Addison-Wesley, Redwood City, 1990).

[5] P. G. O’Shea, M. Reiser, R. A. Kishek, S. Bernal, H. Li, M. Pruessner, V. Yun, Y. Cui, W. Zhang, Y. Zou, et al., Nucl. Instrum. and Methods A 464, 646 (2001).

[6] N. Kjærgaard and M. Drewsen, Phys. Plasmas 8, 1371 (2001).

[7] A. B. Sefkow and R. C. Davidson, Phys. Rev. ST Accel. Beams 9, 090101 (2006).

[8] R. L. Gluckstern, in Proceedings of the 1970 Proton Linear Accelerator Conference (National Accelerator Laboratory, Batavia, IL, 1971), p. 811.

[9] J. Struckmeier and I. Hofmann, Part. Accel. 39, 219 (1992).

[10] N. Brown and M. Reiser, Phys. Plasmas 2, 965 (1995).

[11] R. C. Davidson and C. Chen, Part. Accel. 59, 175 (1998).

[12] R. C. Davidson, Phys. Rev. Lett. 81, 991 (1998).

[13] P. H. Stoltz, R. C. Davidson, and W. W. Lee, Phys. Plasmas 6, 298 (1999).

[14] L. Friedland, Phys. Fluids B 4, 3199 (1992).

[15] L. Friedland, Phys. Plasmas 5, 645 (1998).

[16] R. C. Davidson, H. Qin, and G. Shvets, Phys. Plasmas 7, 1020 (2000).

[17] E. P. Gilson, R. C. Davidson, P. C. Efthimion, R. Majeski, and H. Qin, in Proceedings of the 2003 Particle Accelerator Conference (IEEE Catalog No. 03CH37423C, 2003), p. 2655.

[18] E. P. Gilson, R. C. Davidson, P. C. Efthimion, R. Majeski, and E. A. Startsev, American Institute of Physics Conference Proceedings 692, 211 (2003).

[19] E. P. Gilson, R. C. Davidson, P. C. Efthimion, and R. Majeski, Phys. Rev. Lett. 92, 155002 (2004).

[20] E. P. Gilson, M. Chung, R. C. Davidson, P. C. Efthimion, R. Majeski, and E. A. Startsev, Nucl. Instrum. and Methods A 544, 171 (2005).

[21] E. P. Gilson, M. Chung, R. C. Davidson, M. Dorf, P. C. Efthimion, and R. Majeski, Phys. Plasmas 13, 056705 (2006).

[22] E. P. Gilson, M. Chung, R. C. Davidson, M. Dorf, D. Grote, P. C. Efthimion, R. Majeski, and E. A. Startsev, Nucl. Instrum. and Methods A 577, 117 (2007).

[23] M. Chung, E. P. Gilson, M. Dorf, R. C. Davidson, P. C. Efthimion, and R. Majeski, Phys. Rev. ST Accel. Beams 10, 014202 (2007). 
[24] M. Chung, E. P. Gilson, M. Dorf, R. C. Davidson, P. C. Efthimion, and R. Majeski, Phys. Rev. ST Accel. Beams 10, 064202 (2007).

[25] H. Okamoto and H. Tanaka, Nucl. Instrum. and Methods A 437, 178 (1999).

[26] N. Kjærgaard, K. Mølhave, and M. Drewsen, Phys. Rev. E 66, 015401 (2002).

[27] R. Takai, H. Enokizono, K. Ito, Y. Mizuno, K. Okabe, and H. Okamoto, Jap. J. Appl. Phys. 45, 5332 (2006).

[28] W. Paul and H. Steinwedel, Z. Naturforsch. A 8, 448 (1953). 